\title{
Testosteron im Alter - ein Update
}

\author{
Testosterone in old age - an up-date
}

\author{
Autoren \\ A. Hilbert-Walter ${ }^{1}$ R. Büttner ${ }^{2}$ C. Sieber ${ }^{1,3}$ C. Bollheimer ${ }^{3}$ \\ Institut \\ ${ }^{1}$ Klinik für Innere Medizin 2 - Geriatrie des Klinikums Nürnberg, Nürnberg \\ 2 Innere Medizin, Krankenhaus Bogen \\ 3 Institut für Biomedizin des Alterns, Friedrich-Alexander-Universität Erlangen-Nürnberg
}

\section{Androgendefizit - ein laborchemisch geprägter Begriff}

Eine der Menopause vergleichbare Andropause gibt es nicht. Während es bei der Frau im Alter zwischen 40 und 55 Jahren physiologischerweise zu einem definitiven Erlöschen der ovariellen Östradiol- und Progesteron-Produktion kommt, nimmt beim Mann die Testosteronproduktion mit zunehmendem Alter allenfalls graduell $\mathrm{ab}$ $[15,16]$. Ungeachtet großer interindividueller Schwankungen kommt es damit statistisch zu einer Linksverschiebung des altersspezifischen Referenzbereichs für das Gesamttestosteron [27] ( Abb.1). Ein Androgendefizit wird jedoch altersunabhängig an der Untergrenze des Referenzbereiches für Gesamttestosteron bei jungen Männern festgemacht [4]. Uneinigkeit herrscht dabei über den konkreten Schwellenwert, der zwischen 230 und $345 \mathrm{ng} / \mathrm{dl}$ (entsprechend 8-12 nmol/l) angesetzt wird. Demnach würden bei einem (in praxi gängigen) Schwel- lenwert von $300 \mathrm{ng} / \mathrm{dl}(10,4 \mathrm{nmol} / \mathrm{l})$ bereits etwa $30 \%$ aller über 80 -jährigen Männer der Definition eines laborchemischen Androgendefizits genügen [19].

Im Plasma ist knapp die Hälfte des Gesamttestosterons an Sexualhormon-bindendes Globulin (SHBG) gebunden und damit biologisch inaktiv. Das übrige Plasmatestosteron ist weitgehend an Albumin gebunden und damit auch nur bedingt biologisch aktiv. Nur etwa 2\% des Plasmatestosterons ist nicht Protein-gebunden und entspricht damit dem biologisch aktiven - freien - Testosteron. Analyseverfahren, die selektiv entweder das sogenannte biologisch verfügbare Testosteron (= Albumin-gebundenes plus freies Testosteron) oder nur das freie Testosteron messen, sind für den klinischen Routinebetrieb methodisch zu hinterfragen $[36,37]$. Es gilt aber formell auch ein freier Testosteronspiegel von weniger als 65 $\mathrm{pg} / \mathrm{ml}$ (entsprechend $<225 \mathrm{pmol} / \mathrm{l}$ ) als hinrei-

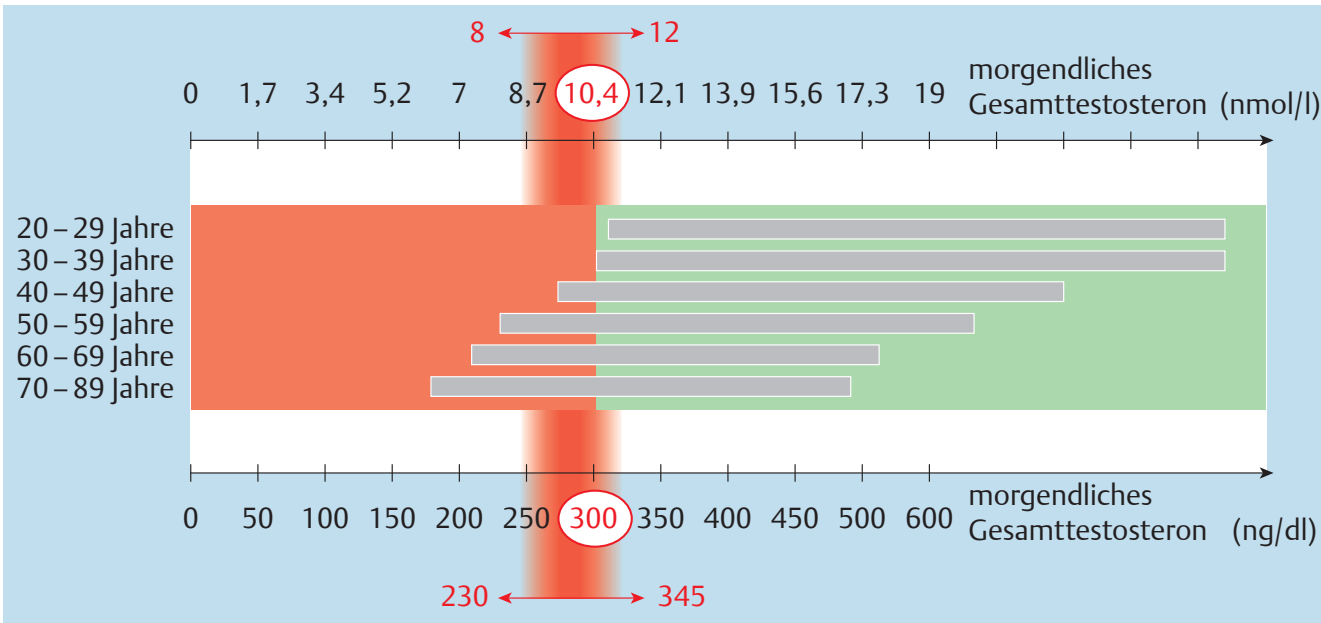

Abb. 1 Der Begriff Androgendefizit ist laborchemisch definiert und orientiert sich an der unteren Grenze des Referenzbereichs jüngerer Männer. Die hellblauen Balken kennzeichnen die nach Schatz et al. [27] altersadjustierten Referenzbereiche.
Urologie, Geriatrie

Schlüisselwörter

Testosteron

Androgendefizit

Late-onset-Hypogonadismus

Gebrechlichkeit

Sarkopenie

Keywords

testosterone

androgen deficiency

late-onset-hypogonadism

frailty

sarcopenia

eingereicht 18.04.2012

akzeptiert 17.07.2012

Bibliografie

DOI 10.1055/s-0032-1305332

Dtsch Med Wochenschr 2012;

137: 2117-2122 - (c) Georg

Thieme Verlag KG · Stuttgart . New York · ISSN 0012-0472

\section{Korrespondenz}

Prof. Dr. med. Cornelius Bollheimer

Institut für Biomedizin des Alterns, Friedrich-AlexanderUniversität Erlangen-Nürnberg Heimerichstraße 58 90419 Nürnberg Tel. 0911/3000511 Fax 0911/3000525 eMail cornelius.bollheimer@ iba.fau.de 
chend für die Diagnose eines laborchemischen Androgendefizits [4]. Hiervon wäre dann rund die Hälfte aller über 80-jährigen Männer betroffen [19].

Bei der Präanalytik einer Testosteronbestimmung sind generell die Tageszeitenschwankungen mit einer Differenz der Werte um bis zu $25 \%$ bei morgendlich-nüchterner versus nachmittäglicher Blutabnahme zu berücksichtigen. Auch wenn die tageszeitliche Varianz im fortgeschrittenen Alter weniger stark ausgeprägt ist, sollte auch beim älteren Mann durch eine morgendlich-nüchterne Blutentnahme (zwischen 7.00 und $11.00 \mathrm{Uhr}$ ) der zirkadiane Spitzenwert für die Diagnose eines Androgendefizits zugrunde gelegt werden [6].

Zusammengefasst erscheint also beim älteren Mann der laborchemische Befund eines Androgendefizits, wie er in den Leitlinien definiert wird [4], ziemlich häufig. Nicht nur in der sogenannten Anti-Aging-Medizin, sondern auch in der Geriatrie stellt sich deshalb die Frage nach der therapeutischen Konsequenz.

\section{kurzgefasst}

Der Befund des Androgendefizits orientiert sich am Referenzbereich für junge Männern. Die hierfür gängigen Schwellenwerte für Gesamttestosteron ( $<300 \mathrm{ng} / \mathrm{dl}$ bzw. $<10,4 \mathrm{nmol} / \mathrm{l}$ ) oder freies Testosteron ( $<65 \mathrm{pg} / \mathrm{ml}$ bzw. $<225 \mathrm{pmol} / \mathrm{l})$ sind relativ hoch und beim Mann in der dritten (60-79) und vierten Lebensphase (> 80 Jahre) nicht hinreichend für die Indikationsstellung einer Testosteronsupplementation.

\section{Androgendefizit - ein vermeidbares Mortalitätsrisiko?}

In zahlreichen Studien wurden niedrige Testosteronwerte des älteren Mannes mit erhöhtem Mortalitätsrisiko in Zusammenhang gebracht $[30,31,33,38]$. Diese Erkenntnisse, die sich beispielsweise auch in einer jüngsten metaanalytischen Auswertung widerspiegeln [1], basieren allerdings auf Observationsstudien. Eine Scheinkorrelation ist aufgrund eines Konfundierungseffektes nicht ausgeschlossen und daher kann auch keine Kausalität abgeleitet werden. Entsprechend könnte ein niedriger Testosteronspiegel beim älteren Mann nur Surrogatparameter für einen schlechteren Gesundheitszustand und die assoziierte erhöhte Mortalität ohne unmittelbaren Kausalitätszusammenhang sein. Gleiches gilt auch für die kürzlich erschienenen Metaanalysen, die einen assoziativen Zusammenhang zwischen niedrigem Testosteronspiegel einerseits und metabolischem Syndrom, Typ-2-Diabetes-mellitus sowie kardiovaskulärer Morbidität andererseits nahelegen [7, 9, 10,24]. Da ebenso hier überzeugende, eine potenzielle Kausalität belegende Interventionsstudien fehlen, sind jegliche praktisch-klinische Schlussfolgerungen verfrüht [25].

\section{kurzgefasst}

Die gefundenen assoziativen Zusammenhänge zwischen niedrigem Testosteron einerseits und Mortalität, kardiovaskulärer Morbidität sowie metabolischen Störungen andererseits belegen [noch] keinen Kausalitätsbezug und sind insofern nicht hinreichend für die Indikationsstellung einer Testosteronsupplementation.

\section{Late-onset-Hypogonadismus - Krankheitsbild von geriatrischer Relevanz?}

Unabhängig von den eben erwähnten kardiometabolischen Implikationen wird dem Testosterondefizit im höheren Lebensalter seit langem ein andrologischer Krankheitswert beigemessen und nosologisch als sogenannter Late-onset-Hypogonadismus beschrieben $[4,28,39]$. Dieser ist dabei ein sowohl klinisch als auch laborchemisch definiertes Syndrom und damit - zumindest theoretisch - nicht gleichzusetzen mit dem laborchemisch definierten Androgendefizit, das formell nur einen Teilbefund des Late-onset-Hypogonadismus darstellt.

In den internationalen Empfehlungen zur Diagnostik und Therapie, die in den Jahren 2008/2009 gleichlautend in 6 unterschiedlichen Fachjournalen publiziert wurden (z.B. [39]), werden klinisch die immanente Verschlechterung der Lebensqualität und die mögliche Beeinträchtigung von Organfunktionen bei Late-onset-Hypogonadismus betont. Die für die Diagnose relevanten Symptome sind in diesen Empfehlungen sehr breit gefächert, wobei neben (i.) sexualanamnestischen Aspekten (Libidoverlust, verminderte Erektionsfähigkeit, Veränderung der Körperbehaarung) auch (ii.) Stimmungsauffälligkeiten (Depressivität, Schlafstörungen) und (iii.) eine Abnahme der körperlichen Fitness (inklusive Abnahme von Knochen- und Muskelmasse sowie Zunahme der Fettmasse) berücksichtigt werden können. In Anbetracht dieser unspezifischen Symptomenpalette mit breitem Deutungsspielraum erscheint der laborchemische Nachweis eines Testosteronmangels mehr oder weniger als der einzig harte Befund bei der Diagnosefindung, womit auch die o.g. Unterscheidung zwischen Androgendefizit und Late-onset-Hypogonadismus akademisch wird.

Nach den internationalen Leitlinien wird eine Testosteronsubstitution (i.) bei Vorliegen stark erniedrigter Gesamttestosteronspiegel ( $<250 \mathrm{ng} / \mathrm{dl}$ bzw. $<8 \mathrm{nmol} / \mathrm{l}$ ) oder (ii.) bei Vorliegen eines moderat erniedrigten Gesamttestosterons (<350 ng/dl bzw. $<12 \mathrm{nmol} / \mathrm{l}$ ) bei zugleich erniedrigtem freien Testosteronspiegel (<65 pg/ml bzw. $<225$ pmol/l) empfohlen [39].

Vor dem Hintergrund der im Jahre 2010 publizierten EMAS-Studie (European Male Aging Study) erscheinen diese Leitlinienempfehlungen allerdings überholt und revisionsbedürftig [40]: An ungefähr 3500 Männern im Alter zwischen 40 und 79 Jahren wurde das für Late-onset-Hypogonadismus typische Symptomenspektrum der Vita sexualis, der Psyche und der körperlichen Fitness durch einen ausführlichen Fragenkatalog unterfüttert. Die anschließende detaillierte statistische Analyse erbrachte das ernüchternde Ergebnis, dass letztlich nur ein Zusammentreffen der drei sexualanamnestischen Symptome (i.) Libidoverlust, (ii.) Impotentia coeundi und (iii.) Ausbleiben der morgendlichen Gliedtumeszenz $(<1 \times /$ Monat $)$ in Kombination mit niedrigem Gesamttestosteron ( $<320 \mathrm{ng} / \mathrm{dl} \mathrm{bzw.}<11 \mathrm{nmol} / \mathrm{l})$ und niedrigem freien Testosteron $(<65 \mathrm{pg} / \mathrm{ml}$ bzw. $<225$ $\mathrm{pmol} / \mathrm{l})$ die klinisch-laborchemische Syndromdiagnose eines Late-onset-Hypogonadismus rechtfertigt ( Abb.2). Legt man diese strikten Diagnosekriterien zugrunde, wäre immerhin etwa jeder 20. Mann über 70 Jahre von einem Late-onset-Hypogonadismus betroffen [15,35]. Als Folge der EMAS-Studie ist mittlerweile die proaktive Grundtendenz bei Diagnose und Therapie, wie sie sich noch in den o.g. internationalen Empfehlungen aus den Jahren 2008/2009 findet [39], einer eher vorsichtig zurückhaltenden Behandlungsstrategie gewichen [17, 29]. 


\begin{tabular}{|c|c|c|c|}
\hline $\begin{array}{l}\text { mindestens } 3 \text { sexual- } \\
\text { anamnestische Symptome }\end{array}$ & Plus & $\begin{array}{l}\text { Laborbefund } \\
\left(7^{00}-11^{00}\right)\end{array}$ & $\begin{array}{l}\text { Abb.2 Der Begriff Late-onset-Hypogonadis- } \\
\text { mus bezeichnet ein sowohl klinisch als auch } \\
\text { laborchemisch definiertes Syndrom. Die ehe- }\end{array}$ \\
\hline $\begin{array}{l}\text { Libidoabnahme } \\
\text { und } \\
\text { (mehrheitlich) Impotentia coeundi } \\
\text { und } \\
\text { Ausbleiben morgendlicher } \\
\text { Gliedtumeszenz }\end{array}$ & & $\begin{array}{l}\text { Gesamttestosteron } \\
<320 \mathrm{ng} / \mathrm{dl}(\approx 11 \mathrm{nmol} / \mathrm{l}) \\
\quad \text { und } \\
\text { Freies Testosteron } \\
<65 \mathrm{pg} / \mathrm{ml}(\approx 225 \mathrm{pmol} / \mathrm{l})\end{array}$ & $\begin{array}{l}\text { nostische Symptomenliste muss vor dem Hin- } \\
\text { tergrund der EMAS-Studie [40] gekürzt wer- } \\
\text { den und sich auf die sexualmedizinische Ana- } \\
\text { mnese konzentrieren. Der Late-onset- } \\
\text { Hypogonadismus verliert damit an geriatri- } \\
\text { scher Bedeutung. }\end{array}$ \\
\hline
\end{tabular}

\section{kurzgefasst}

Der Late-onset-Hypogonadismus ist ein bislang eher überdiagnostiziertes sexualmedizinisch akzentuiertes Syndrom des rüstigen älteren Mannes und besitzt keine unmittelbare geriatrische Relevanz.

\section{Testosteron - ein Medikament der Geriatrie?}

Während das revidierte Krankheitskonzept des Late-onset-Hypogonadismus eher der Lifestyle- und Anti-Aging-Medizin zugeordnet werden muss, wird jedoch der Testosteronmangel seit längerem auch unter spezifisch geriatrischem Fokus erforscht.

Anfangs interessierte dabei besonders die „anti-osteoporogene“ Wirkung von Testosteron, die vornehmlich indirekt, d.h. durch Umwandlung (Aromatisierung) von Testosteron zum direkt knochenaktiven Östradiol mediiert wird $[11,22]$. So war in einer großen Beobachtungsstudie an rund 2500 über 65-Jährigen ein stark erniedrigtes Gesamttestosteron ( $<200 \mathrm{ng} / \mathrm{dl}$ bzw. $<6,4$ nmol/l) im Vergleich zu normalen Testosteron-Spiegeln $(>400$ ng/dl bzw. > 13,9 nmol/l) mit einem etwa 3,5-fach höheren Osteoporoserisiko (sogenannte messtechnische Osteoporose mit T-Wert $_{\text {Hüfte }} \leq-2,5$ ) behaftet [13]. Dieselbe Studie legte allerdings auch nahe, dass das beim älteren Mann viel häufiger vorkommende moderate Androgendefizit (Gesamttestosteron 200 - $400 \mathrm{ng} / \mathrm{dl}$ bzw. 6,4 - 13,9 nmol/l) nicht mit einem erhöhten Osteoporoserisiko einhergeht [13]. Folgerichtig ergab eine Metaanalyse, die den Einfluss einer Testosteronsupplementation auf den Knochen untersuchte und dabei Studien an älteren Männern miteinbezog, keine Verbesserung der Knochendichte [34]. Vor diesem Hintergrund verwundert es schließlich nicht, dass der Testosteronspiegel beim älteren Mann keine Vorhersagekraft für osteoporotisch bedingte Frakturen besitzt [22]. Lediglich bei Stürzen, die zusammen mit dem osteologischen Befund das Risiko einer peripheren osteoporotischen Fraktur ausmachen, zeigte sich in einer Beobachtungsstudie eine mögliche Gefahrenkonstellation durch verminderte Testosteronspiegel. So hatten Männer mit einem Durchschnittsalter von $71 \pm 5$ Jahren und einem Androgendefizit (Gesamttestosteron entsprechend niedrigster Quartile: $<377 \mathrm{ng} / \mathrm{dl}$ bzw. $<13,1 \mathrm{nmol} / \mathrm{l}$ ) ein rund 4fach höheres Sturzrisiko im Vergleich zu Männern mit hohem Testosteronspiegel (Gesamttestosteron entsprechend höchster Quartile der Kohorte: > 568 ng/dl bzw. > 19,7 nmol/l) [5].

Losgelöst vom Problemkreis Osteoporose/Sturz/Fraktur orientieren sich die geriatrisch geprägten Studien zu Testosteron in letzter Zeit zunehmend an den operationalisierten Begriffen Frailty (engl. „Gebrechlichkeit“) und „Sarkopenie“, d.h. einer Abnahme von Muskelmasse und -kraft. Auf der Ebene von Beobachtungsstudien ist der Zusammenhang zwischen (dem Risiko für) Frailty und einem Androgendefizit allerdings nicht komplett überzeugend: Wenn überhaupt, gibt es einen solchen nur für das freie Testosteron $[8,18,21,23]$. Im Jahre 2010 erschienen drei randomisiert-kontrollierte Interventionsstudien, die im Zusammenhang mit Frailty und Sarkopenie den Nutzen und das Risiko einer Testosteronsupplementation näher untersuchten $[3,20,32,35]$ :

- In der ersten Studie von Srinivas-Shankar et al. [32] wurden rund 275 Männer im Alter über 65 Jahre (Durchschnittsalter 74 Jahre) aufgenommen, die ein Androgendefizit aufwiesen (Gesamttestosteron $<12 \mathrm{nmol} / \mathrm{l}[\approx 375 \mathrm{ng} / \mathrm{dl}]$ und/oder freies Testosteron $<250 \mathrm{pmol} / 1[\approx 70 \mathrm{pg} / \mathrm{ml}]$ ) und mindestens ein sogenanntes Fried-Kriterium [14] erfüllten. Die Fried-Kriterien bilden den biomedizinisch-phänotypischen Rahmen von Frailty und umfassen dabei (i.) einen subjektiv empfundenen Erschöpfungszustand, (ii.) einen ungewollten Gewichtsverlust, (iii.) eine Abnahme der Muskelkraft, (iv.) eine Verlangsamung der Gehgeschwindigkeit sowie (v.) eine Abnahme körperlicher Aktivität und des damit verbundenen Kalorienverbrauchs [14]. Bei dieser Studiengruppe zeigten sich nach 6monatiger Testosteronsupplementation (25-75 mg transdermales Testosteron) in der Verumgruppe Positiveffekte hinsichtlich (i.) der Muskelkraft der unteren Extremität (isokinetische Kraftwerte bei der Kniestreckung), (ii.) der Körperzusammensetzung (fettfreie Masse $\uparrow /$ Fettmasse $\downarrow$ ) und (iii.) der Lebensqualität. Insgesamt waren diese Effekte jedoch relativ gering, sodass trotz Signifikanz die Relevanz dieser Effekte hinterfragt werden kann. Darüber hinaus erscheint die isokinetische Kraftmessung der Kniestrecker als primärer Outcome-Parameter eklektizistisch, insbesondere vor dem Hintergrund der multiplen Testung diverser weiterer muskuloskelettaler Parameter mit Negativresultaten.

- In der Studie von Kenny et al. [20] wurden rund 130 Männer im Alter über 57 Jahre (Durchschnittsalter 77 Jahre) mit Androgendefizit (u.a. Gesamttestosteronspiegel < $350 \mathrm{ng} / \mathrm{dl}[\approx 12$ $\mathrm{nmol} / \mathrm{l}]$ ) aufgenommen. Die Rekrutierungspraxis dieser Studie ist verwirrend: Sie beinhaltete ursprünglich Männer im Alter über 50 Jahre mit osteoporotisch bedingter Hüftfaktur. Diese Einschlusskriterien wurden im Laufe der Studie umgeändert, in Hinblick auf (i.) Altersgrenze ( $\geq 60$ Jahre), (ii.) osteologische Kriterien (entweder T-Score-Hüfte $\leq-2,0$ oder osteoporotisch bedingte Hüftfraktur innerhalb der letzten 5 Jahre) sowie (iii.) Frailty ( $\geq 1$ Fried-Kriterium [14]). Nach einjähriger transdermaler Testosteronsupplementation zeigte sich gegenüber Placebo ein signifikanter Nutzen nur im Hinblick auf die Körperzusammensetzung (fettfreie Masse $\uparrow$ / Fettmasse $\downarrow$ ); die muskuloskelettalen Funktionsparameter verbesserten 


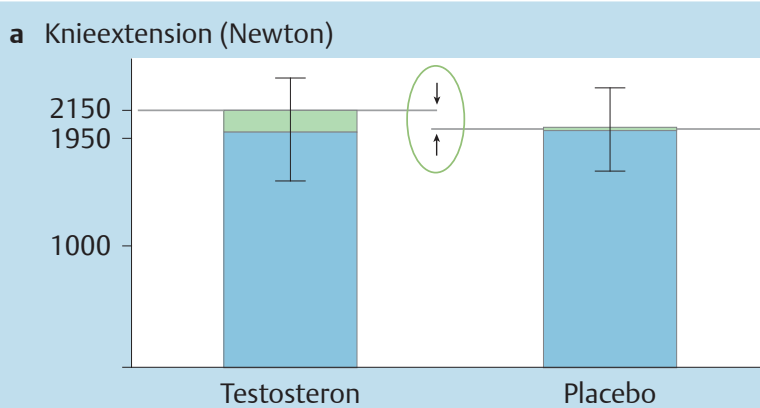

b (Prozent innerhalb der Gruppe)

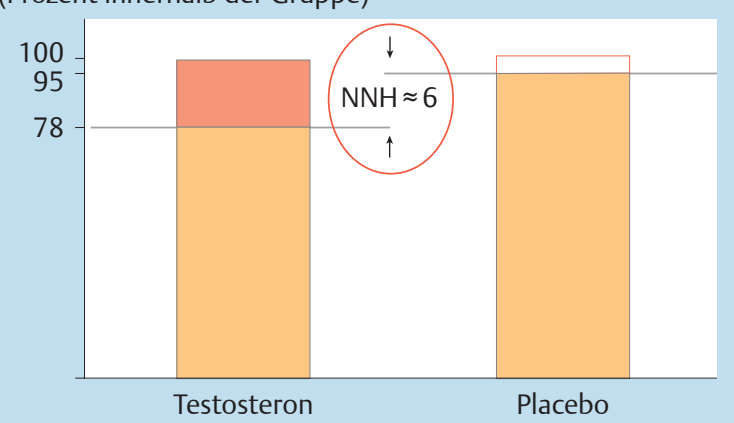

Studienteilnehmer in der Verumgruppe mit $\geq 1$ kardiovaskulären Ereignis

$\square$ Studienteilnehmer in der Placebogruppe mit $\geq$ kardiovaskulärem Ereignis

Studienteilnehmer ohne kardiovaskuläres Ereignis

Abb.3 Ernüchternde und zugleich aufrüttelnde Ergebnisse der TOM-Studie [35]. Männer mit Mobilitätsbeeinträchtigung und Androgendefizit, im Durchschnitt 74 Jahre, erhielten in einer randomisiert-kontrollierten Studie Testosteron. Der geringfügige Testosteron-Effekt beim primären Outcome-Parameter (Kraft der unteren Extremität [Kniestreckung], Graph 3a) wurde durch eine starke Zunahme kardiovaskulärer Ereignisse erkauft (Graph 3b). NNH = number needed to harm.

sich nicht. Eine Unterscheidung zwischen primären und sekundären Outcome-Parametern wurde bei dieser methodisch angreifbaren Studie gar nicht in Betracht gezogen.

- Zu erheblichen Diskussionen führte schließlich auch die sogenannte TOM-Studie (Testosterone in Older Men with Mobility Limitations) aus der Arbeitsgruppe um Shalender Bhasin $[3,35]$. In dieser Studie wurden rund 200 Männer im Alter über 65 Jahre (Durchschnittsalter 74 Jahre) aufgenommen, die ein Androgendefizit (Gesamttestosteron $<12 \mathrm{nmol} / \mathrm{l}$ $[\approx 375 \mathrm{ng} / \mathrm{dl}]$ und/oder freies Testosteron $<155 \mathrm{pmol} / \mathrm{l}$ $[\approx 50 \mathrm{pg} / \mathrm{ml}]$ ) aufwiesen und in ihrer Mobilität beeinträchtigt waren. Die Studie war initial auf ein halbes Jahr ausgelegt; die Intervention bestand in der transdermalen Applikation von 50-150 mg Testosteron täglich. Allerdings musste die Studie wegen Häufung kardiovaskulärer Ereignisse in der Verumgruppe vorzeitig abgebrochen werden. Bei den Probanden, die bis dahin mindestens eine 3-monatige Intervention durchlaufen hatten, zeigten sich durch Testosteron allenfalls minimale - gleichwohl signifikante - Verbesserungen in den isometrisch gemessenen Kraftwerten für die Knieextension (,leg press“, primärer Endpunkt) und das Bankdrücken („,chest press“) sowie ferner beim Treppensteigen mit Gewichten und bei der Körperzusammensetzung (fettfreie Masse $\uparrow$ ) (O Abb.3).
Auf dem Boden dieser drei Interventionsstudien muss man zu dem ernüchternden Ergebnis kommen, dass die Positiveffekte einer (alleinigen) Testosteronsupplementation bei gebrechlichen Männern vernachlässigbar gering sind. Unter Einbeziehung einer weiteren, morphologisch ausgerichteten Interventionsstudie [2] kann durch Testosteronsupplementation bei Frailty-gefährdeten Männern allenfalls eine Zunahme der Muskelmasse erwartet werden, die sich aber funktionell kaum auszahlt.

Fairerweise sollten diese ernüchternden Ergebnisse zu Testosteron im geriatrischen Bereich wissenschaftlich zunächst nur als Zwischenergebnis gewertet werden. So kann es durchaus sein, dass Testosteron zwar nicht allein, jedoch synergistisch im Rahmen eines kombinierten Therapieansatzes von Nutzen sein könnte [26]. Allerdings muss zuvor unbedingt die aktuell am heftigsten diskutierte Problematik geklärt werden, welche das potenzielle intrinsisch-kardiovaskuläre Risiko einer Testosteronsupplementation im Alter betrifft.

\section{kurzgefasst}

Geriatrisch akzentuierte Interventionsstudien mit Testosteron existieren im Zusammenhang mit Knochendichte sowie Muskelmasse/-kraft. Sie tangieren damit die Entitäten Osteoporose und Sarkopenie, überzeugen jedoch in beiden Fällen nicht.

\section{Testosteronsupplementation im Alter - eine Pandorabüchse? \\ $\nabla$}

Die bereits oben erwähnte, hochrangig publizierte TOM-Studie [3] erzielte ihre Aufmerksamkeit nicht aufgrund ihrer bescheidenen Ergebnisse im Hinblick auf die Testosteron-bedingte Verbesserung der Muskelfunktion, sondern wegen des in der Verumgruppe gehäuften Auftretens kardiovaskulärer Ereignisse. Das absolute Risiko entsprach - je nach gewählter Kategorisierung - einer number needed to harm $(\mathrm{NNH})$ zwischen 5,5 und 11 , sodass die eigentlich auf 6 Monate ausgelegte Studie vorzeitig abgebrochen werden musste.

Die kardiovaskulären Komplikationen in der TOM-Studie kamen völlig unerwartet und stehen überdies im Gegensatz zur eingangs erwähnten Assoziation zwischen kardiovaskulärem Risiko und Androgendefizit. Ferner erschien noch kurz vor Veröffentlichung der TOM-Studie eine entwarnende Metaanalyse, die sich mit den kardiovaskulären und Prostata-spezifischen Nebenwirkungen einer mindestens 3 Monate andauernden Testosterontherapie auseinandersetzte [12]. Die Autoren dieser Metaanalyse wiesen nur auf einen Testosteron-bedingten Abfall des HDL-Cholesterins und einen Anstieg des Hämatokrits hin, der sich in der Subgruppenanalyse bei den Über-65-Jährigen besonders stark zeigte [12]. Im Nachhinein könnte sich dahinter ein Erklärungsansatz für die unerwarteten Ergebnisse der TOMStudie verbergen (Blutviskositätsproblem).

Theoretisch könnten die Ergebnisse der TOM-Studie aufgrund der relativ kleinen Fallzahl und/oder aufgrund der ungleichmäßigen Komorbiditätsverteilung bei arterieller Hypertonie und Hyperlipidämie auch zufällig bzw. Testosteron-unabhängig zustande gekommen sein. Dennoch sind die Daten existent und lassen sich nicht so leicht wegdiskutieren, sodass bei der Ver- 


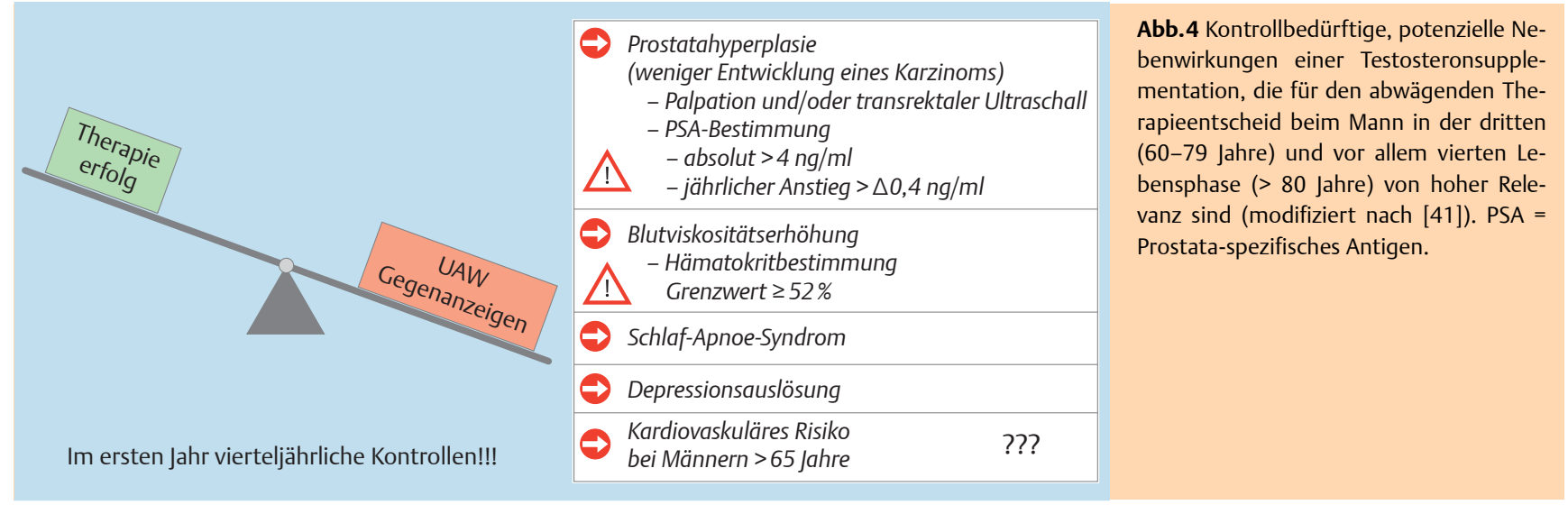

ordnung von Testosteron zum jetzigen Zeitpunkt zur besonderen Vorsicht geraten werden muss. Vor dem Hintergrund der TOM-Studie und ungeachtet der (vielleicht überinterpretierten?) Assoziationsstudien zwischen Androgendefizit und kardiovaskulärem Risikoprofil plädieren wir deshalb beim älteren Mann mit kardiovaskulärer Anamnese für eine streng zurückhaltende Verordnung von Testosteron. Folgerichtig sollte eine Behandlung nur als wohlbegründete Einzelfallentscheidung entweder nach fachärztlich endokrinologischer Maßgabe oder im Rahmen überwachter klinischer Studien erfolgen. In diesem Falle müssen dann auch die übrigen potenziellen Nebenwirkungen einer Testosteronsupplementation bedacht und entsprechend engmaschig kontrolliert werden ( Abb.4).

\section{kurzgefasst}

Dem Studienabbruch der sog. TOM-Studie lag das vermehrte Auftreten kardiovaskulärer Ereignisse bei Testosteron-supplementierten älteren Männern zugrunde. Diese unerwartete und bislang ungeklärte Nebenwirkung sollte eine wohlüberlegte und streng restriktive Neuverordnung von Testosteron beim Mann ab 65 Jahren bedingen.

\section{Fazit für Klinik und Praxis}

- Ein Androgendefizit ist ein laborchemischer Befund und begründet beim älteren Mann noch keine hinreichende Indikation für eine Testosteronsupplementation.

- Assoziative Daten bringen niedrigere Testosteronspiegel mit einem erhöhten kardiometabolischen Morbiditäts- und Mortalitätsrisiko in Verbindung. Eine Kausalität allerdings ist bislang nicht nachgewiesen, sodass sich hieraus keine unmittelbare Konsequenz auf die Verordnungspraxis von Testosteron ergibt.

Das bisherige klinische Konzept des Late-onset-Hypogonadismus (LOH) birgt aufgrund seiner weichen klinischen Klassifikationskriterien die Gefahr einer Überdiagnose und Überbehandlung. Die vor dem Hintergrund aktueller Daten enggefasste Definition des LOH als strikt sexualmedizinisches Syndrom mit Androgendefizit ist deshalb zu begrüßen.

- Eine Testosteronsupplementation aus geriatrischer Indikation ist falsch. Dagegen sprechen die insgesamt enttäuschenden Effekte im muskuloskelettalen Bereich und die ungeklärte Frage nach kardiovaskulären Nebenwirkungen.
Autorenerklärung: Die Autoren erklären, dass sie keine finanziellen Verbindungen mit einer Firma haben, deren Produkt in diesem Artikel eine wichtige Rolle spielt (oder mit einer Firma, die ein Konkurrenzprodukt vertreibt).

\section{Literatur}

1 Araujo AB, Dixon JM, Suarez EA et al. Clinical review: Endogenous testosterone and mortality in men: a systematic review and metaanalysis. J Clin Endocrinol Metab 2011; 96: 3007-3019

2 Atkinson RA, Srinivas-Shankar U, Roberts SA et al. Effects of testosterone on skeletal muscle architecture in intermediate-frail and frail elderly men. J Gerontol A Biol Sci Med Sci 2010; 65: 1215-1219

3 Basaria S, Coviello AD, Travison TG et al. Adverse events associated with testosterone administration. N Engl J Med 2010; 363: 109122

4 Bhasin S, Cunningham GR, Hayes FJ et al. Testosterone therapy in men with androgen deficiency syndromes: an Endocrine Society clinical practice guideline. J Clin Endocrinol Metab 2010; 95: 25362559

5 Bischoff-Ferrari HA, Orav EJ, Dawson-Hughes B. Additive benefit of higher testosterone levels and vitamin D plus calcium supplementation in regard to fall risk reduction among older men and women. Osteoporos Int 2008; 19: 1307-1314

6 Brambilla DJ, Matsumoto AM, Araujo AB et al. The effect of diurnal variation on clinical measurement of serum testosterone and other sex hormone levels in men. J Clin Endocrinol Metab 2009; 94: 907913

7 Cattabiani C, Basaria S, Ceda GP et al. Relationship between testosterone deficiency and cardiovascular risk and mortality in adult men. J Endocrinol Invest 2012; 35: 104-120

8 Cawthon PM, Ensrud KE, Laughlin GA et al. Sex hormones and frailty in older men: the osteoporotic fractures in men (MrOS) study. J Clin Endocrinol Metab 2009; 94: 3806-3815

9 Corona G, Monami M, Rastrelli $G$ et al. Type 2 diabetes mellitus and testosterone: a meta-analysis study. Int J Androl 2011; 34: 528540

10 Corona G, Rastrelli G, Vignozzi L et al. Testosterone, cardiovascular disease and the metabolic syndrome. Best Pract Res Clin Endocrinol Metab 2011; 25: 337-353

11 Falahati-Nini A, Riggs BL, Atkinson EJ et al. Relative contributions of testosterone and estrogen in regulating bone resorption and formation in normal elderly men. J Clin Invest 2000; 106: 1553-1560

12 Fernández-Balsells MM, Murad MH, Lane $M$ et al. Clinical review 1: Adverse effects of testosterone therapy in adult men: a systematic review and meta-analysis. J Clin Endocrinol Metab 2010; 95: 25602575

13 Fink HA, Ewing SK, Ensrud KE et al. Association of Testosterone and Estradiol Deficiency with Osteoporosis and Rapid Bone Loss in Older Men. J Clin Endocrinol Metab 2006; 91: 3908-3915

14 Fried LP, Tangen CM, Walston J et al. Frailty in older adults: evidence for a phenotype. J Gerontol A Biol Sci Med Sci 2001; 56: 146-156 
15 Gray A, Feldman HA, McKinlay JB et al. Age, disease, and changing sex hormone levels in middle-aged men: results of the Massachusetts Male Aging Study. J Clin Endocrinol Metab 1991; 73: 10161025

16 Harman SM, Metter EJ, Tobin JD et al. Baltimore Longitudinal Study of Aging. Longitudinal effects of aging on serum total and free testosterone levels in healthy men. Baltimore Longitudinal Study of Aging. J Clin Endocrinol Metab 2001; 86: 724-731

17 Huhtaniemi I, Forti G. Male late-onset hypogonadism: pathogenesis, diagnosis and treatment. Nat Rev Urol 2011; 8: 335-344

18 Hyde Z, Flicker L, Almeida OP et al. Low free testosterone predicts frailty in older men: the health in men study. J Clin Endocrinol Metab 2010; 95: 3165-3172

19 Kaufman JM, Vermeulen A. The decline of androgen levels in elderly men and its clinical and therapeutic implications. Endocr Rev 2005; 26: 833-876

20 Kenny AM, Kleppinger A, Annis $K$ et al. Effects of transdermal testosterone on bone and muscle in older men with low bioavailable testosterone levels, low bone mass, and physical frailty. J Am Geriatr Soc 2010; 58: 1134-1143

21 Krasnoff JB, Basaria S, Pencina MJ et al. Free testosterone levels are associated with mobility limitation and physical performance in community-dwelling men: the Framingham Offspring Study. J Clin Endocrinol Metab 2010; 95: 2790-2799

22 Mellström D, Vandenput L, Mallmin H et al. Older men with low serum estradiol and high serum SHBG have an increased risk of fractures. J Bone Miner Res 2008; 23: 1552-1560

23 Mohr BA, Bhasin S, Kupelian V et al. Testosterone, sex hormone-binding globulin, and frailty in older men. J Am Geriatr Soc 2007; 55: 548-555

24 Ruige JB, Mahmoud AM, De Bacquer D et al. Endogenous testosterone and cardiovascular disease in healthy men: a meta-analysis. Heart 2011; 97: 870-875

25 Saad F, Aversa A, Isidori AM et al. Testosterone as potential effective therapy in treatment of obesity in men with testosterone deficiency: a review. Curr Diabetes Rev 2012; 8: 131-143

26 Sattler F, Bhasin S, He J et al. Testosterone threshold levels and lean tissue mass targets needed to enhance skeletal muscle strength and function: the HORMA trial. J Gerontol A Biol Sci Med Sci 2011; 66: 122-129

27 Schatzl G, Madersbacher S, Temml C et al. Serum androgen levels in men: impact of health status and age. Urology 2003; 61: 629-633

28 Seftel AD, Miner MM, Kloner RA et al. Office evaluation of male sexual dysfunction. Urol Clin North Am 2007; 34: 463-482

29 Shelton JB, Rajfer J. Androgen deficiency in aging and metabolically challenged men. Urol Clin North Am 2012; 39: 63-75
30 Shores MM, Matsumoto AM, Sloan KL et al. Low serum testosterone and mortality in male veterans. Arch Intern Med 2006; 166: 16601665

31 Shores MM, Moceri VM, Gruenewald DA et al. Low testosterone is associated with decreased function and increased mortality risk: a preliminary study of men in a geriatric rehabilitation unit. J Am Geriatr Soc 2004; 52: 2077-2081

32 Srinivas-Shankar $U$, Roberts SA, Connolly MJ et al. Effects of testosterone on muscle strength, physical function, body composition, and quality of life in intermediate-frail and frail elderly men: a randomized, double-blind, placebo-controlled study. J Clin Endocrinol Metab 2010; 95: 639-650

33 Tivesten A, Vandenput L, Labrie F et al. Low serum testosterone and estradiol predict mortality in elderly men. J Clin Endocrinol Metab 2009; 94: 2482-2488

34 Tracz MJ, Sideras K, Boloña ER et al. Testosterone use in men and its effects on bone health. A systematic review and meta-analysis of randomized placebo-controlled trials. J Clin Endocrinol Metab 2006; 91: 2011-2016

35 Travison TG, Basaria S, Storer TW et al. Clinical meaningfulness of the changes in muscle performance and physical function associated with testosterone administration in older men with mobility limitation. J Gerontol A Biol Sci Med Sci 2011; 66: 1090-1099

36 Vermeulen A, Kaufman JM, Giagulli VA. Influence of some biological indexes on sex hormone-binding globulin and androgen levels in aging or obese males. J Clin Endocrinol Metab 1996; 81: 18211826

37 Vermeulen A, Verdonck L, Kaufman JM. A critical evaluation of simple methods for the estimation of free testosterone in serum. J Clin Endocrinol Metab 1999; 84: 3666-3672

38 Vikan T, Schirmer H, Njølstad I et al. Endogenous sex hormones and the prospective association with cardiovascular disease and mortality in men: the Tromsø Study. Eur J Endocrinol 2009; 161: 435442

39 Wang $C$, Nieschlag $E$, Swerdloff $R$ et al. Investigation, treatment and monitoring of late-onset hypogonadism in males: ISA, ISSAM, EAU, EAA and ASA recommendations. Eur J Endocrinol 2008; 159: $507-$ 514

40 Wu FC, Tajar A, Beynon JM et al. Identification of late-onset hypogonadism in middle-aged and elderly men. N Engl J Med 2010; 363: 123-135

41 Zitzmann M. Die Therapie des Hypogonadismus des Mannes. Internist (Berl.) 2008; 49: 559-569 